\title{
PinXI suppresses tumorigenesis by negatively regulating telomerase/telomeres in colorectal carcinoma cells and is a promising molecular marker for patient prognosis
}

\author{
This article was published in the following Dove Press journal: \\ OncoTargets and Therapy \\ 3 August 2016 \\ Number of times this article has been viewed
}

Dong Qian,' Jingjing

Cheng,' Xiaofeng Ding,' Xiuli Chen,' Xi Chen,' 'Yong Guan,' Bin Zhang, ${ }^{2}$ Jiefu Wang, ${ }^{3}$ Puchun Er,' Minghan Qiu,' Xianliang Zeng,' Yihang Guo,' Huanhuan Wang,' Lujun Zhao,' Dan Xie, ${ }^{4}$ Zhiyong Yuan,' Ping Wang,' Qingsong Pang'

'Department of Radiotherapy, ${ }^{2}$ Department of Lung Cancer, ${ }^{3}$ Department of Colorectal Cancer, Tianjin Medical University Cancer Institute and Hospital, National Clinical Research Center for Cancer, Key Laboratory of Cancer Prevention and Therapy, Tianjin, ${ }^{4}$ State Key Laboratory of Oncology in South China, Cancer Center, Sun Yat-Sen University, Guangzhou, People's Republic of China
Correspondence: Qingsong Pang Department of Radiotherapy, Tianjin Medical University Cancer Institute and Hospital, National Clinical Research Center for Cancer, Key Laboratory of Cancer Prevention and Therapy, Huanhu West Street, Tianjin 300060,

People's Republic of China

Tel $+86222334 \quad$ I405

Fax +86 222334 |405

Email pangqingsong2013@163.com
Abstract: PinX1 plays positive and negative roles in the maintenance of telomerase and telomeres, as well as in tumorigenesis. The aim of the present study was to investigate the expression and clinical significance of PinX1 in colorectal carcinoma (CRC) and to determine the effect of PinX1 on CRC cell proliferation and apoptosis. A total of $86 \mathrm{CRC}$ patients treated with radical resection and 5-fluorouracil-based adjuvant chemotherapy were enrolled in this study. The expression dynamics of PinX1 was detected by immunohistochemistry in the CRC patients and 25 normal colonic mucosa controls. PinX1 expression was significantly reduced in tumor tissues as compared to normal tissues, and the rate of PinX1 protein low/negative expression in CRC and normal tissues was $60 \%(52 / 86)$ and $24 \%(6 / 25)$, respectively $(P=0.037)$. In addition, PinX1 downregulation was significantly associated with short overall survival $(P=0.016)$ and disease-free survival $(P=0.042)$ in CRC patients. Cox proportional hazards model further revealed that PinX1 expression was an independent factor in predicting overall survival and disease-free survival for CRC patients. Furthermore, we demonstrated that ectopic overexpression of PinX1 in CRC cells inhibited their proliferation, promoted apoptosis, repressed telomerase activity, and induced telomere shortening. These findings suggest that PinX1 may be a prognostic biomarker for CRC patients' survival and that it inhibits cell proliferation and promotes apoptosis by repressing telomerase activity and inducing telomere shortening. Targeting PinX1 may therefore provide a novel therapeutic strategy for CRC patients.

Keywords: colorectal carcinoma, PinX1, cell proliferation, apoptosis, telomerase, telomere, prognostic biomarker

\section{Introduction}

Colorectal carcinoma (CRC) is the third most common type of cancer and the second leading cause of cancer deaths worldwide. ${ }^{1,2}$ The incidence of CRC has increased annually, together with lifestyle changes, and is ranked the fifth most commonly diagnosed cancer in the People's Republic of China. ${ }^{3}$

$\mathrm{CRC}$ arises through a multistep carcinogenic process during which cells acquire the hallmarks of cancer, such as immortality. ${ }^{4,5}$ This involves the stabilization of telomere length, which is achieved by telomerase activation in over $85 \%$ of human tumors. ${ }^{6-8}$ Telomerase is a specialized ribonucleoprotein reverse transcriptase required for the de novo synthesis of telomeric DNA at chromosomal ends after the cell cycle. It is repressed in the majority of somatic cells, but is reactivated in most human cancer types, and these malignant cells maintain their telomere lengths and demonstrate unlimited proliferative 
capacity. ${ }^{9,10}$ Thus, the maintenance of telomeres by telomerase constitutes part of CRC tumorigenesis and could be a promising target for predicting clinical prognosis and cancer therapy.

The nuclear protein Pin2-TRF1 interacting protein (PinX1) was originally shown to be a telomerase/telomere interacting factor, which is evolutionary conserved from yeasts to humans. Zhou and $\mathrm{Lu}^{11}$ found that PinX1 bound the telomerase catalytic protein subunit telomerase reverse transcriptase, and the PinX1-telomerase reverse transcriptase interaction has been experimentally shown to have a telomere activity-inhibitory effect, suggesting it is an intrinsic telomerase inhibitor and a putative tumor suppressor. Other groups demonstrated that $\mathrm{Pin} X 1^{-/}$mice are embryonic lethal, while PinX1 ${ }^{+-}$mice spontaneously develop malignant tumors resulting from chromosomal instability. ${ }^{12}$

Our most recent in vitro and in vivo studies revealed that the ectopic overexpression of PinX1 in telomerase-positive cancer cells resulted in resistance to DNA damaging agents and ionizing radiation, which could be attributed to the maintenance of telomere stability, and the reduction of DNA damage-induced cell apoptosis and mitosis catastrophe. ${ }^{13-15}$ Furthermore, the high expression of PinX1 positively correlated with esophageal squamous cell carcinoma patient resistance to chemoradiotherapy and was a strong predictor for short disease-specific survival in esophageal squamous cell carcinoma patients. ${ }^{15}$ However, Deng et al reported that PinX1 expression was decreased in CRC and that its downregulation was significantly associated with poorer 5 -year overall survival (OS) and disease-free survival (DFS) rates. ${ }^{16}$ These contradictory results suggested that PinX1 abnormalities may be tumor type-specific and could play different roles in cancer progression at different stages.

In this study, we investigated PinX1 regulation in CRC patients and examined its effect on CRC cell apoptosis, proliferation, and telomere maintenance. Our findings highlight the functions and roles of PinX1 in CRC and suggest PinX1 may be used as a novel prognostic marker and potential therapeutic target for CRC patients.

\section{Materials and methods}

\section{Tissue specimens and cell cultures}

CRC tissue samples were obtained with written informed consent under Institutional Review Board-approved protocols. All paraffin-embedded samples were collected at the Tianjin Medical University Cancer Institute and Hospital (Tianjin, People's Republic of China). The CRC cases selected were based on clear pathological diagnosis and follow-up data and were from patients who had not received previous local or systemic treatment in the period 2006-2010. Patients consisted of 52 males and 34 females, with a median age of 53 (range, 37-76) years. Tumor stage was conducted according to the 2010 tumor node metastasis classification of malignant tumors by the American Joint Committee on Cancer, and patients were at stages I $(n=6)$, II ( $\mathrm{n}=27)$, III $(\mathrm{n}=45)$, and IV $(\mathrm{n}=8)$. Cellular differentiation was graded according to the World Health Organization (WHO) grading system. In addition, 25 normal colonic mucosa tissues removed during benign colorectal lesions surgical procedures were obtained from the same period. All samples were formalin-fixed, paraffin-embedded, and pathologically diagnosed. This study was approved by the Institute Research Ethics Committee of Tianjin Medical University Cancer Institute and Hospital.

The human colorectal cancer cell lines SW1116 and SW480 were obtained from ATCC (American Type Culture Collection, Manassas, VA, USA). Cells were cultured $<3$ months after resuscitation and maintained in RPMI 1640 media (SW1116) or Dulbbecco's Modified Eagle's Medium, (SW480) (Thermo Fisher Scientific, Waltham, MA, USA) with $10 \%$ (vol/vol) fetal bovine serum (Thermo Fisher Scientific) at $37^{\circ} \mathrm{C}$ in a $5 \% \mathrm{CO}_{2}$ incubator.

\section{Immunohistochemistry}

Immunohistochemistry (IHC) staining was performed on $5 \mu \mathrm{m}$ tissue sections rehydrated through graded alcohols. Endogenous peroxidase activity was blocked with $0.3 \%$ hydrogen peroxide for 15 minutes. An antigen retrieval process was accomplished in a microwave oven with $10 \mathrm{mM}$ citrate buffer, $\mathrm{pH}$ 6.0, for 15 minutes. Nonspecific binding was blocked with $10 \%$ normal rabbit serum for 10 minutes. The tissue slides were incubated with the rabbit polyclonal antibody against human full-length PinX1 (ProteinTech Group, Inc., Rosemont, IL, USA; 1:100 dilution) overnight at $4^{\circ} \mathrm{C}$. Subsequently, the slides were sequentially incubated with biotinylated goat antirabbit immunoglobulin (ZSGBBIO, Beijing, People's Republic of China) at a concentration of $1: 100$ for 30 minutes at $37^{\circ} \mathrm{C}$ and then reacted with a streptavidin-peroxidase conjugate for 30 minutes at $37^{\circ} \mathrm{C}$ and $3^{\prime}-3^{\prime}$ diaminobenzidine as a chromogen substrate. The nucleus was counterstained using Meyer's hematoxylin. Known immunostaining-positive/negative slides were used as positive and negative controls. Two independent observers blinded to the clinicopathologic information performed scoring using a previously validated scoring system for PinX1 expression. ${ }^{17}$ This system scores nuclear PinX1 expression by recording the percentage of nuclei staining positive for 
the PinX1 protein, irrespective of staining intensity, in which PinX1 immunoreactivity was classified into two groups: low expression, when PinX1-positive cells were $<50 \%$, including samples with negative expression of PinX1 (Figure 1B and $\mathrm{C}$ ), and high expression, when at least $50 \%$ of the cells showed positive immunoreactivity of PinX1 in the nuclei (Figure 1A and D). In an IHC study, a minimum of 500 epithelial cells was counted for each normal or tumor case.

\section{Plasmid construction, lentivirus production, and transduction}

The full-length PinX1 constructs were amplified and cloned into the pCDH-CMV-MSC-EF1-coGFP cDNA expression lentivector (System Biosciences, Palo Alto, CA, USA). Then, the lentiviral expression construct and packaging plasmid mix were cotransfected into 293 cells to generate recombinant lentivirus according to the manufacturer's manual. SW1116
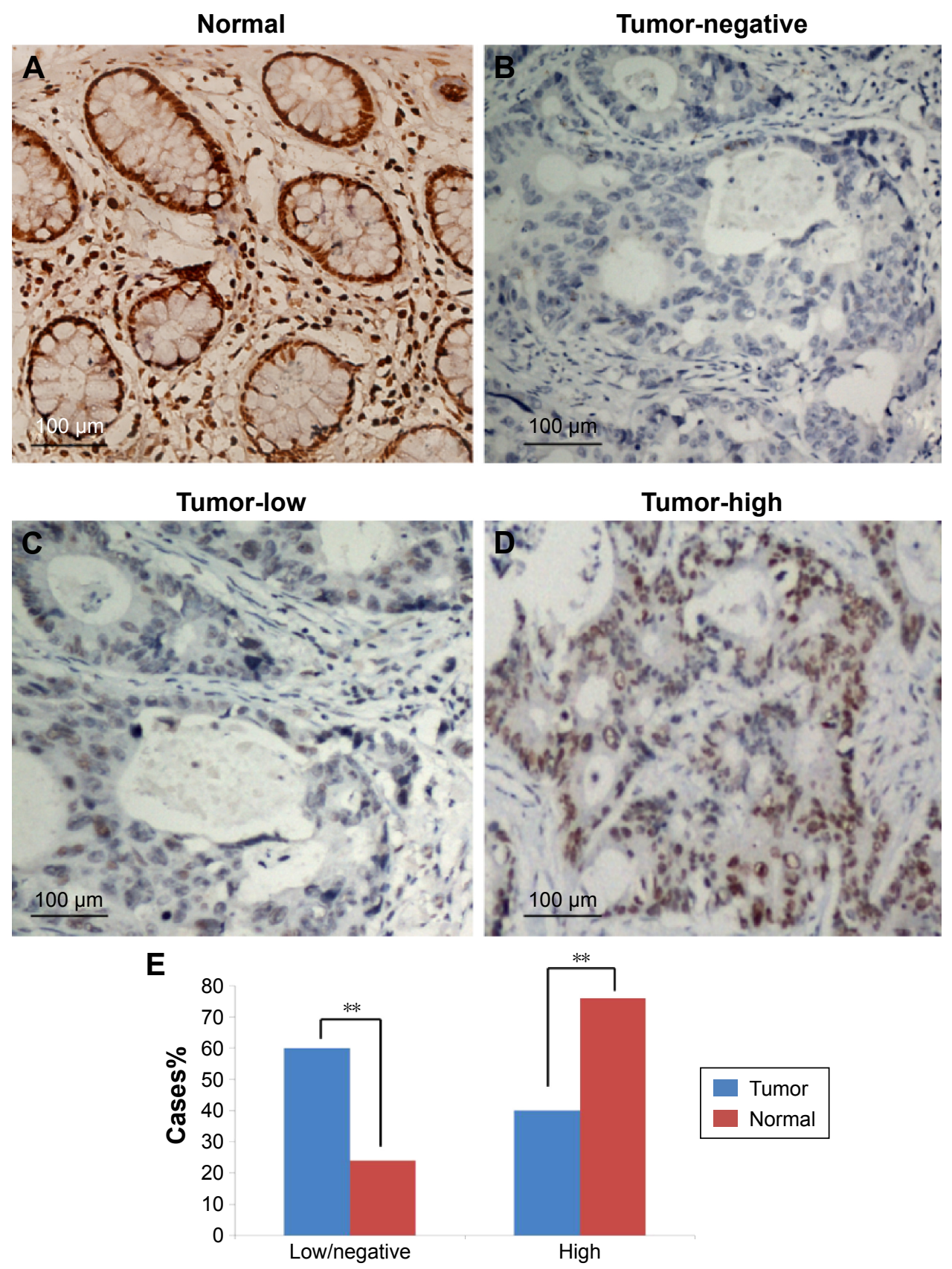

Figure I Expression of PinXI in human colorectal carcinoma (CRC) tissues.

Notes: (A) High expression of PinXI was observed in a normal colonic mucosa tissue, in which almost all colonic mucosa cells showed positive staining of PinXI in nuclei. (B) A CRC (case II) shows negative expression of PinXI. (C) A CRC (case 52) was examined with low expression of PinXI, in which <50\% of carcinoma cells showed positive staining of PinXI in nuclei. (D) High expression of PinXI was examined in another CRC sample (case 36 ) in which $>50 \%$ carcinoma cells demonstrated positive staining of PinXI. (E) The percentage of the cases with low/negative or high PinXI expression in tumor and normal tissues $(* * P<0.01)$. 
and SW480 cells were infected with recombinant lentivirustransducing units plus $8 \mu \mathrm{g} / \mathrm{mL}$ Polybrene (Sigma-Aldrich Co., St Louis, MO, USA).

\section{Western blotting assay}

Cells were lysed in lysis buffer. BCA kit (Pierce, Rockford, IL, USA) was used to determine protein concentrations. SDS-PAGE and Western blotting were done according to standard procedures. Proteins were detected with antibodies recognizing full-length PinX1 (45 kDa). GAPDH (Santa Cruz Biotechnology, Inc., Dallas, TX, USA) was used as a loading control.

\section{MTT proliferation assay}

Cell viability was measured with the use of 3-(4, 5-dimethylthiazol-2-yl)-2, 5-diphenyl tetrazolium bromide (MTT) proliferation assay (Sigma-Aldrich Co.). Briefly, cells were seeded in 96-well plates and cultured. Cell viability was examined following standard procedures. Experiments were done in triplicate.

\section{Annexin V-APC/propidium iodide flow cytometry apoptosis assay}

Annexin V-APC and propidium iodide stains were used to determine the percentage of cells undergoing apoptosis. The apoptosis assay was conducted using the protocol supplied by the manufacturer (BD Biosciences, San Jose, CA, USA). Each sample was then subjected to analyses by flow cytometry (BD FACSCanto II Flow Cytometer, BD Biosciences).

\section{Telomerase activity assay}

Telomerase activity was measured with the TRAPeze telomerase detection kit (Chemicon, Temecula, CA, USA). PCR products were separated by electrophoresis on a $12.5 \%$ nondenaturing polyacrylamide gel, visualized by SYBR green (Thermo Fisher Scientific) staining and semiquantitated according to the manufacturer's instruction. Briefly, telomerase activity consists of the intensity of the TRAP product band and the processivity of TRAP ladders.

\section{Telomere lengths analysis}

Two micrograms of genomic DNA from tissue extracts were doubly digested with Hinf I and Rsa I overnight at $37^{\circ} \mathrm{C}$. The DNA products of enzymes digestion were electrophoresed on $0.8 \%$ agarose gel and transferred onto a nylon membrane for hybridization with digosin-labbed (TTAGGG)3 oligos. The hybridization signal was detected by the AP-conjugated antidigosin antibodies (Hoffman-La Roche Ltd., Basel, Switzerland) and imaged by CDP-Star (Hoffman-La Roche Ltd.).

\section{Statistical analysis}

Statistical analysis was carried out using SPSS 16.0 (SPSS Standard version 13.0, SPSS Inc., Chicago, IL, USA). Student's $t$-test was used to analyze the results expressed as mean \pm standard deviation. The chi-squared test or Fisher's exact test was used to analyze the association of PinX1 expression and clinicopathological parameters. The survival curves were plotted by Kaplan-Meier analysis. Differences were considered significant when the $P$-value was $<0.05$.

\section{Results}

\section{PinXI was downregulated in CRC tissues}

We examined PinX1 protein expression using IHC in 86 cases of primary $\mathrm{CRC}$ and 25 normal colonic mucosa controls (Figure 1A-D). Low/negative PinX1 expression was observed in 60\% (negative: 9 ; low: $43,52 / 86$ ) of CRC tissues and only $24 \%$ (negative: 0 ; low: $6,6 / 25$ ) of normal colonic mucosa. High PinX1 expression was observed in 40\% (34/86) of CRC tissues but 76\% (19/25) of normal colonic mucosa $(P=0.012$, Figure 1E). The association between PinX1 expression and clinicopathological features of the 86 $\mathrm{CRC}$ cases is summarized in Table 1. PinX1 expression correlated closely with tumor differentiation $(P=0.037)$, but no significant association was found between PinX1 expression and other clinicopathological variables, such as patient age, WHO grade, tumor location, or tumor size $(P>0.05)$.

\section{Low expression of PinXI correlates with poor CRC survival}

Univariate analysis found that low PinX1 expression was significantly correlated with poor OS (Figure $2 \mathrm{~A}, P=0.016$ ) and DFS (Figure 2B, $P=0.042$ ) in CRC patients. Multivariate analysis showed that PinX1 expression as well as tumor node metastasis stage, local invasion, and lymph node metastasis were independent predictors of patient OS and DFS ( $P=0.015,0.006,0.031$, and 0.014 , respectively; Table 2$)$.

\section{PinXI suppresses proliferation and promotes apoptosis in CRC cells}

To explore the potential cancer-suppressive role of PinX1 in $\mathrm{CRC}$, the PinX1 construct was stably transfected into the SW1116 and SW480 cell lines. PinX1 expression was confirmed by Western blotting (Figure 3A), and the MTT assay showed that ectopic overexpression of PinX1 inhibited cell growth compared with blank and vector-transfected control cells (Figure 3B, $* * P<0.01$ ). To determine whether PinX1 has a proapoptotic effect on CRC cells, the Annexin $\mathrm{V} /$ propidium iodide staining cell apoptosis assay was performed. Flow cytometry analysis then indicated that 
Table I Clinicopathological correlation of PinXI expression in colorectal carcinomas

\begin{tabular}{|c|c|c|c|c|}
\hline Variables & Cases & Low/negative expression (\%) & High expression (\%) & $P$-value* \\
\hline Age (years) & & & & 0.612 \\
\hline$\leq 55^{* *}$ & 40 & $24(60)$ & $16(40)$ & \\
\hline$>55$ & 46 & $28(60.8)$ & $18(39.1)$ & \\
\hline Sex & & & & 0.164 \\
\hline Male & 52 & $34(65.3)$ & $18(34.6)$ & \\
\hline Female & 34 & $18(52.9)$ & $16(47.1)$ & \\
\hline Tumor location & & & & 0.431 \\
\hline Proximal & 24 & $13(54.2)$ & II (45.8) & \\
\hline Distal & 27 & 17 (62.9) & $10(37)$ & \\
\hline Rectum & 35 & $22(62.8)$ & $13(37.1)$ & \\
\hline Tumor size $(\mathrm{cm}) * * *$ & & & & 0.540 \\
\hline$\leq 5$ & 36 & $22(6 I . I)$ & $14(38.9)$ & \\
\hline$>5$ & 50 & $30(60.0)$ & $20(40.0)$ & \\
\hline Differentiation & & & & 0.037 \\
\hline Well & 19 & $9(47.3)$ & $10(52.6)$ & \\
\hline Moderate & 39 & $23(59.0)$ & $16(41.0)$ & \\
\hline Poor & 28 & $20(71.4)$ & $8(28.6)$ & \\
\hline Local invasion & & & & 0.415 \\
\hline TI-2 & 35 & $23(65.7)$ & $12(34.3)$ & \\
\hline T3-4 & 51 & $29(56.8)$ & $22(43.1)$ & \\
\hline Lymph metastasis & & & & 0.216 \\
\hline No & 38 & $24(63.1)$ & $14(36.8)$ & \\
\hline Yes & 48 & $28(58.3)$ & $20(4 I .7)$ & \\
\hline Stage & & & & 0.422 \\
\hline I/II & 33 & $19(57.6)$ & $14(42.4)$ & \\
\hline III/IV & 53 & $33(62.6)$ & $20(37.7)$ & \\
\hline
\end{tabular}

Notes: *Chi-square test; **mean age; $* * *$ mean tumor size. Data in bold indicates statistical significance $(P<0.05)$.

PinX1 significantly promoted tumor cell apoptosis in both cells compared with control cells under normal conditions (Figure $3 \mathrm{C}, * * P<0.01$ ).

\section{PinXI suppresses telomerase activity and induces telomere shortening in CRC cells}

PinX1 functions as an intrinsic inhibitor of telomerase enzymatic activity. TRAP assays in our study consistently indicated that the ectopic overexpression of PinX1 significantly suppressed telomerase enzymatic activities in SW1116 cells (approximately fourfold, Figure 4A). SW1116 cells overexpressing PinX1 were also shown to have reduced telomere lengths compared with control vector cells (approximately twofold, Figure 4B). Together, these results demonstrate that PinX1 suppresses telomerase activities and induces telomere shortening in CRC cells.
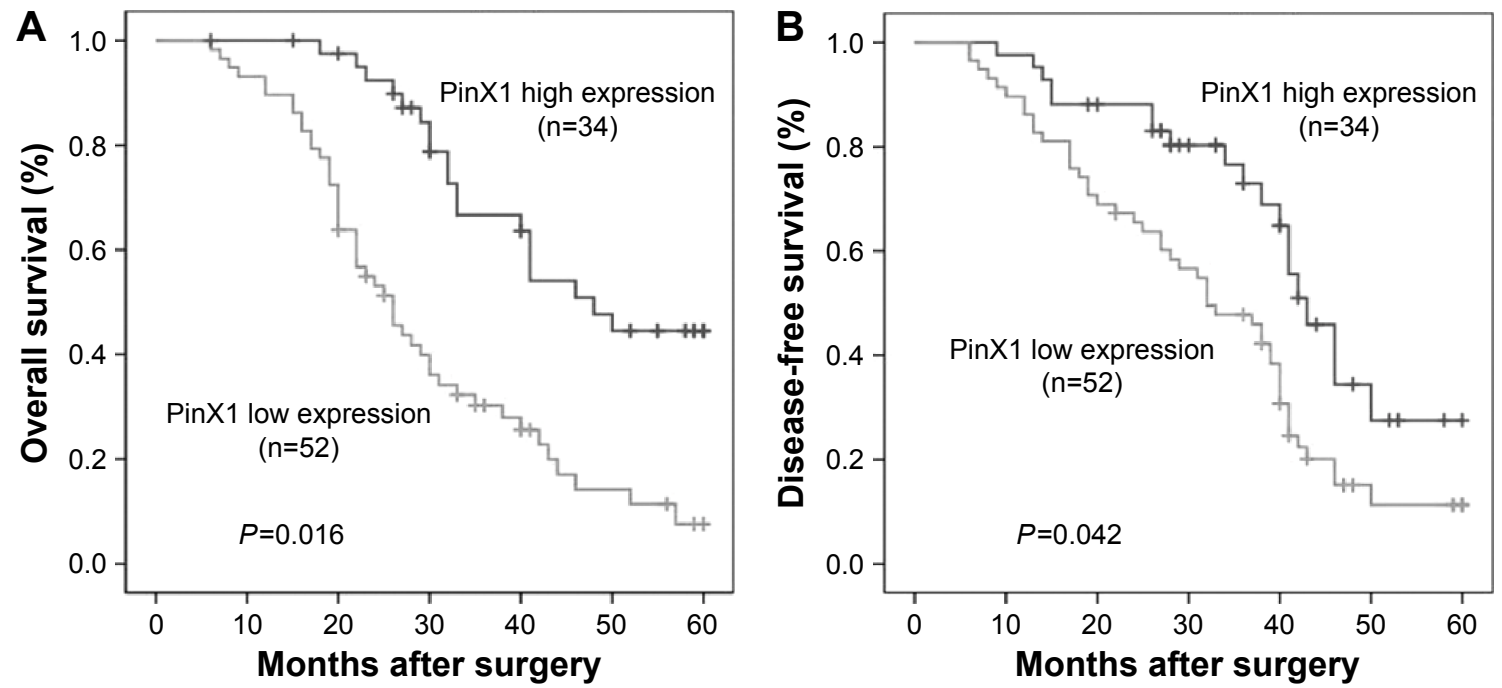

Figure 2 Kaplan-Meier statistical analyses of overall survival (OS) and disease-free survival (DFS) in 86 colorectal carcinoma patients.

Notes: Downregulation of PinXI was significantly associated with poorer OS $(\mathbf{A})$ and poorer DFS (B), according to PinXI expression levels in the primary tumor ( $P<0.05$, log-rank test). 
Table 2 Multivariate Cox regression analysis for OS in CRC patients

\begin{tabular}{llll}
\hline Factors & HR & $\mathbf{9 5 \%} \mathbf{C l}$ & $\boldsymbol{P}$-value \\
\hline PinXI expression & 2.296 & $1.557-4.520$ & 0.015 \\
TNM stage (III/IV vs VS I/II) & 2.973 & $1.429-6.985$ & 0.006 \\
Local invasion (T3-4 vs TI-2) & 1.421 & $0.493-3.247$ & 0.031 \\
Lymph metastasis (yes vs no) & 1.310 & $0.652-3.017$ & 0.014 \\
\hline
\end{tabular}

Abbreviations: $\mathrm{Cl}$, confidence interval; $\mathrm{CRC}$, colorectal carcinoma; $\mathrm{HR}$, hazard ratio; OS, overall survival; TNM, tumor node metastasis.

\section{Discussion}

Surgical resection and adjuvant chemotherapy are standard, effective treatments for CRC patients in the early stages or with locally advanced disease. ${ }^{18,19}$ However, CRC patients with the same clinical stage often display considerable variability in their response to these anticancer therapies because of differences in intrinsic molecular characteristics. ${ }^{20}$ Therefore, there is an urgent need to identify subgroups of

A
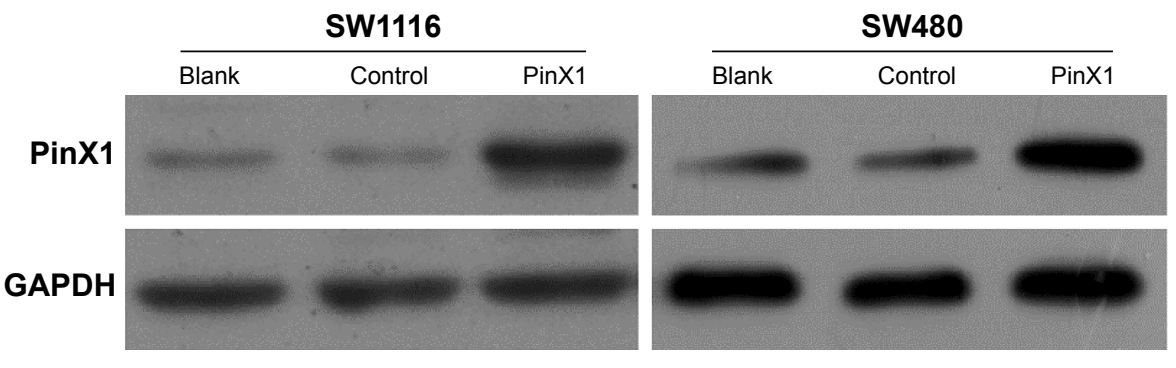

B
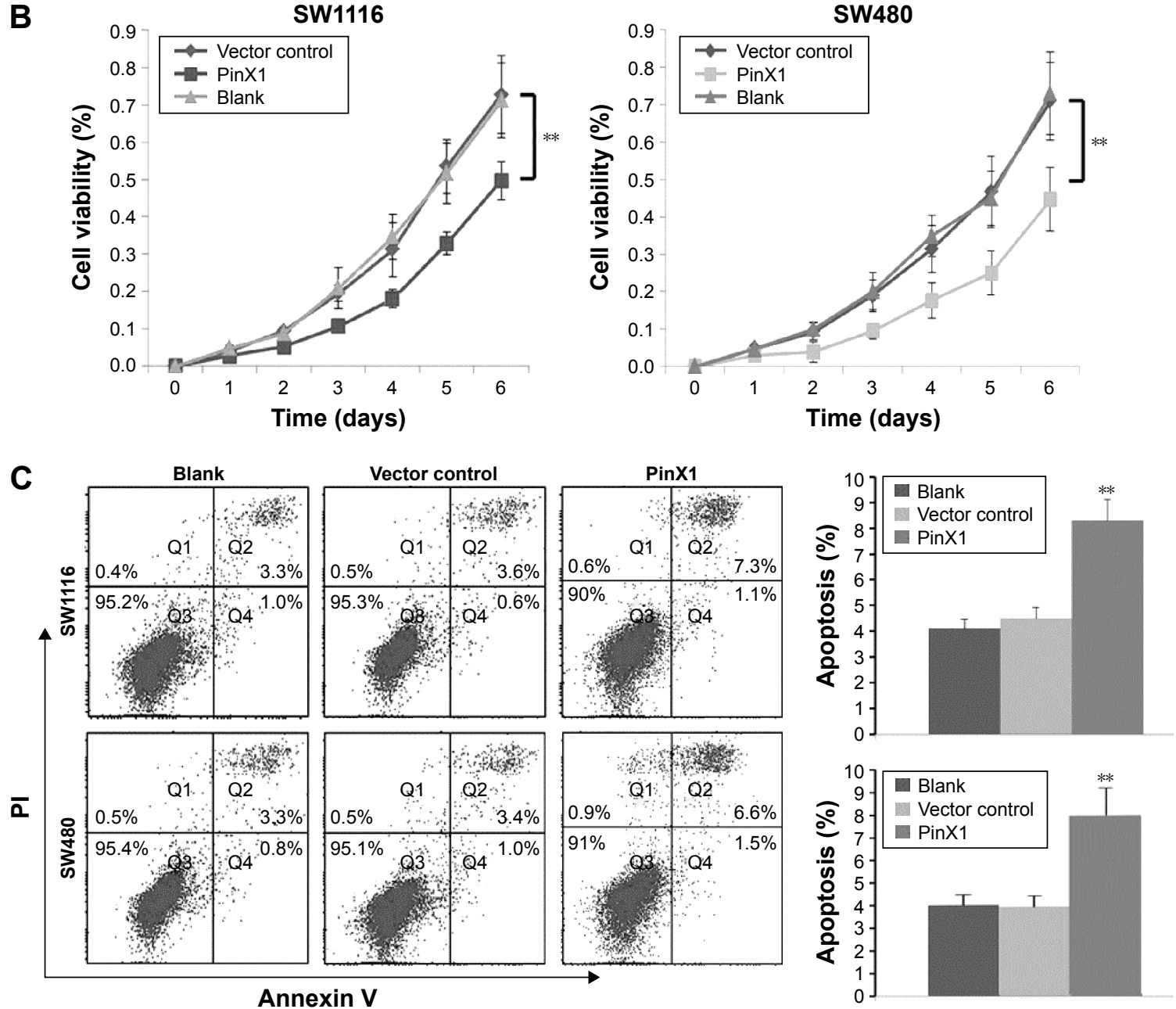

Figure 3 PinXI suppresses proliferation and promotes apoptosis in colorectal carcinoma cells in vitro.

Notes: (A) Expression of PinXI was detected by Western blot in stable transfected SWIII6 and SW480cells (SWIII6-PinXI; SW480-PinXI) relative to empty vector control cells (SWIII6-Vector; SW480-Vector) and blank control cells (SWIII6-Blank; SW480-Blank). Expression was normalized against endogenous GAPDH. (B) Cell growth rate was suppressed by ectopic overexpression of PinXI in SWIII6 cells detected by 3-(4, 5-dimethylthiazol-2-yl)-2, 5-diphenyl tetrazolium bromide assay. Results are expressed as mean \pm standard deviation (SD) of three independent experiments. (C) PinXI promoted tumor cell apoptosis in SWIII6 cells compared with the vectortransfected control cells and blank control cells under normal conditions. Cell apoptotic death events were monitored by Annexin $\mathrm{V} / \mathrm{propidium}$ iodide (PI) staining and flow cytometry assays. The percentage of cell apoptosis was shown as the mean \pm SD from three independent experiments (**P<0.0I, $P$-value was according to Student's $t$-test). 

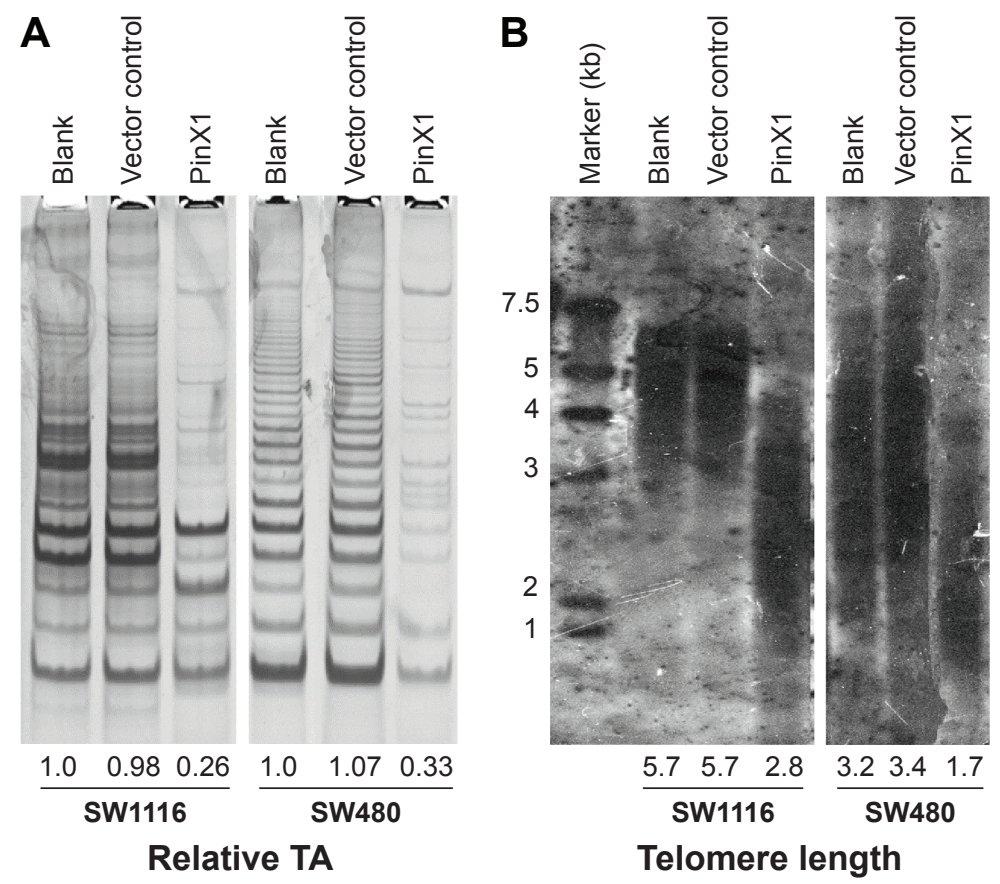

Figure 4 Effect of PinXI on telomerase activity (TA) and telomere length in colorectal carcinoma cells.

Notes: TA was measured by TRAP assays and Southern blot analysis of telomeric terminal restriction fragments was used for the determination of the telomere length. Ectopic overexpression of PinXI in SWIII 6 and SW480 cells decreased TA (A) and shortened telomere (B).

patients with different outcomes to develop individualized treatments. Although CRC has been widely studied, the identification of specific genetic alterations associated with patient outcomes remains limited. ${ }^{21}$ Thus, further work is needed in the development of reliable molecular biomarkers, not only for CRC but also for an array of human neoplasms.

Telomerase has been implicated in human cancers for its role in counteracting the telomere shortening-induced replicative senescence. Targeting pathways involved in the maintenance of telomerase/telomeres may be therefore a useful anticancer therapeutic strategy. ${ }^{22,23}$ Originally, PinX1 was identified as an intrinsic telomerase inhibitor and a putative tumor suppressor through its binding to and inhibition of telomerase. ${ }^{11}$ However, we previously demonstrated that PinX1 had a positive role in regulating telomere maintenance by telomerase and that it inhibited the drug-induced apoptotic effect in telomerase-positive cancer cells, which may be caused by the PinX1-mediated telomerase/telomere recruitment pathway. ${ }^{13,15}$ More recently, Deng et al reported that PinX1 expression was significantly reduced in CRC tissues compared with normal tissues and that its downregulation was significantly associated with adverse 5-year OS and DFS in CRC patients treated with radical resection and 5-fluorouracil-based adjuvant chemotherapy. ${ }^{16}$ However, our studies reported high PinX1 expression in $\sim 60 \%$ of esophageal squamous cell carcinoma patient $(n=157)$ tissues from two cancer centers, but high PinX1 expression in only $16.7 \%$ of normal esophageal mucosa specimens. Further correlation analysis showed that high PinX1 expression was positively correlated with poor disease-specific survival in esophageal squamous cell carcinoma patients treated with chemoradiotherapy. ${ }^{14}$ These contradictory results led us to detect PinX1 expression in CRC tissues from our own hospital.

In accordance with Deng et al's reports, we found that the expression of PinX1 in CRC tissues was lower than in normal noncancerous tissues. ${ }^{16}$ Moreover, low PinX1 expression was an independent predictor for short OS and DFS in CRC patients treated with radical resection and 5-fluorouracil-based adjuvant chemotherapy. We believe that telomerase/telomere pathways play critical roles in cell differentiation and tumorigenesis. ${ }^{24}$ As shown in Table 1, we found that low expression of PinX1 was closely correlated with poor differentiation of $\mathrm{CRC}$, indicating that PinX1 may play roles in tumor progression through regulating telomerase/telomere pathways. In addition, we observed that ectopic overexpression of PinX1 could increase the sensitivity of CRC cells to 5-fluorouracil, the main component of the adjuvant chemotherapy regimen administered to our patients with CRC (Figure S1), which further supports our clinical data and those of Deng et al. ${ }^{16}$ Our results, together with those of others, suggest that PinX1 plays a dual role in cancer progression and that the abnormalities and/or functions of PinX1 in tumorigenesis are complicated and possibly tumor type-specific. 
Cell proliferation and apoptosis are important stages of cancer development and therapy and are regulated by multiple molecular pathways, including telomere maintenance..$^{25-27}$ Our current in vitro results showed that the ectopic restoration of PinX1 suppressed cell proliferation and promoted cellular apoptosis in both SW1116 and SW480 cells. Our previous study demonstrated that upregulation of PinX1 in urothelial carcinoma of the bladder cells increased the proportion of cells in the G0/G1 phase and decreased those in the S phase through the p16/cyclin D1 pathway. ${ }^{28}$ We obtained similar results for SW1116 cells in the present study (Figure S2). These results suggest that PinX1 may have a tumor suppressive function in CRC progression.

Based on our recent work and other previous studies, we propose that $\mathrm{Pin} X 1$ regulates the telomere maintenance of cancer cells through at least two distinct mechanisms. In the first, we postulate that PinX1 induces telomere dysfunction by binding to telomerase and directly suppressing its activity. In the second, we speculate that PinX1 plays a positive role in telomere length regulation through stimulating the access of telomerase to telomeres.

Zhang et a ${ }^{29}$ previously revealed that PinX1 without the G-patch motif (69-328 amino acid) induced cell apoptosis and G1 arrest, but had no effects on telomerase activity in CRC SW480 cells. However, the detailed role of PinX1 in telomerase/telomere regulation has not been clearly elucidated in CRC cells. Herein, we hypothesized whether the reintroduction of full-length PinX1 would regulate telomerase activity and telomere length in CRC cells. Our results clearly showed that telomerase activity is markedly inhibited when the telomere length is dramatically shortened in full-length PinX1-restored SW1116 and SW480 cells. G-patch motif of PinX1 may play critical roles in regulating telomerase activity in CRC cells. Moreover, in our IHC study, a significant inverse correlation of PinX1 expression and hTERT expression was found in our CRC patients (Figure S3). This result was in accordance with previous reports ${ }^{16,17}$ and provides evidence of a telomerase-inhibited function of PinX1 in CRC.

To our knowledge, the maintenance of telomere stability by telomerase is required for the immortalization and rapid growth of almost all cancer cells. ${ }^{30}$ Moreover, telomere dysfunction-induced senescence and/or apoptosis are important mechanisms of tumorigenesis inhibition in humans while the disruption of telomere maintenance can enhance cancer cell sensitivity to chemotherapeutic agents and ionizing radiation. ${ }^{31,32}$ Therefore, our findings strongly suggest that the functional loss of PinX1 leads to enhanced telomerase activity and telomere stabilization. In turn, this promotes cellular proliferation and cellular resistance to apoptosis, which consequently favors CRC progression. Thus, the examination of PinX1 may be used as an effective tool to predict the outcome of CRC patients and optimize the making of clinical decisions.

\section{Conclusion}

In summary, we describe the expression pattern of PinX1 in human CRC. Our results provide a basis for the concept that low PinX1 expression, as revealed by $\mathrm{IHC}$, may be a novel predictor of aggressive CRC and an independent prognostic factor for CRC patients. Furthermore, functional and mechanistic studies suggest that PinX1 inhibits cell proliferation and promotes apoptosis via the suppression of telomerase activity and the shortening of telomere length in CRC cells. Thus, targeting of the PinX1-mediated telomerase/telomere maintenance pathway might represent a novel therapeutic strategy to improve the outcome for CRC patients.

\section{Acknowledgments}

This study was supported by Doctor Supporting Foundation of Tianjin Medical University Cancer Institute and Hospital (No B1302 and B1305), the National Nature Science Foundation of China (No 81401948, 81472182, and 81472797), and Tianjin Municipal Science and Technology Commission (No 15JCQNJC11800 and 15JCYBJC25500). All procedures performed in studies involving human participants were in accordance with the ethical standards of the institutional and/ or national research committee and with the 1964 Declaration of Helsinki and its later amendments or comparable ethical standards. This article does not contain any studies with animals performed by any of the authors.

\section{Disclosure}

The authors report no conflicts of interest in this work.

\section{References}

1. Siegel R, Ma J, Zou Z, Jemal A. Cancer statistics, 2014. CA Cancer J Clin. 2014;64(1):9-29.

2. Siegel R, Desantis C, Jemal A. Colorectal cancer statistics, 2014. $C A$ Cancer J Clin. 2014;64(2):104-117.

3. Chen W, Zheng R, Zhang S, et al. Annual report on status of cancer in China, 2010. Chin J Cancer Res. 2014;26(1):48-58.

4. Markowitz SD, Bertagnolli MM. Molecular origins of cancer: molecular basis of colorectal cancer. $N$ Engl J Med. 2009;361(25):2449-2460.

5. Engelhardt M, Drullinsky P, Guillem J, Moore MA. Telomerase and telomere length in the development and progression of premalignant lesions to colorectal cancer. Clin Cancer Res. 1997;3(11):1931-1941.

6. Kim NW, Piatyszek MA, Prowse KR, et al. Specific association of human telomerase activity with immortal cells and cancer. Science. 1994; 266(5193):2011-2015. 
7. Hahn WC, Counter CM, Lundberg AS, Beijersbergen RL, Brooks MW, Weinberg RA. Creation of human tumour cells with defined genetic elements. Nature. 1999;400(6743):464-468.

8. Patton EE, Harrington L. Cancer: trouble upstream. Nature. 2013; 495(7441):320-321.

9. Hanahan D, Weinberg RA. Hallmarks of cancer: the next generation. Cell. 2011;144(5):646-674.

10. Schmidt JC, Cech TR. Human telomerase: biogenesis, trafficking, recruitment, and activation. Genes Dev. 2015;29(11):1095-1105.

11. Zhou XZ, Lu KP. The Pin2/TRF1-interacting protein PinX1 is a potent telomerase inhibitor. Cell. 2001;107(3):347-359.

12. Simila S, Kokkonen J. Coexistence of celiac disease and Down syndrome. Am J Ment Retard. 1990;95(1):120-122.

13. Zhang B, Bai YX, Ma HH, et al. Silencing PinX1 compromises telomere length maintenance as well as tumorigenicity in telomerase-positive human cancer cells. Cancer Res. 2009;69(1):75-83.

14. Qian D, Zhang B, He LR, et al. The telomere/telomerase binding factor PinX1 is a new target to improve the radiotherapy effect of oesophageal squamous cell carcinomas. J Pathol. 2013;229(5):765-774.

15. Zhang B, Qian D, Ma HH, et al. Anthracyclines disrupt telomere maintenance by telomerase through inducing PinX1 ubiquitination and degradation. Oncogene. 2012;31(1):1-12.

16. Deng W, Jiao N, Li N, Wan X, Luo S, Zhang Y. Decreased expression of PinX1 protein predicts poor prognosis of colorectal cancer patients receiving 5-FU adjuvant chemotherapy. Biomed Pharmacother. 2015; $73: 1-5$.

17. Cai MY, Zhang B, He WP, et al. Decreased expression of PinX1 protein is correlated with tumor development and is a new independent poor prognostic factor in ovarian carcinoma. Cancer Sci. 2010;101(6): 1543-1549.

18. Simillis C, Hompes R, Penna M, Rasheed S, Tekkis PP. A systematic review of transanal total mesorectal excision. Is this the future of rectal cancer surgery? Colorectal Dis. 2016;18(1):19-36.

19. Dienstmann R, Salazar R, Tabernero J. Personalizing colon cancer adjuvant therapy: selecting optimal treatments for individual patients. $J$ Clin Oncol. 2015;33(16):1787-1796.

20. van Geel RM, Beijnen JH, Bernards R, Schellens JH. Treatment individualization in colorectal cancer. Curr Colorectal Cancer Rep. 2015;11(6):335-344.
21. Wang H, Liang L, Fang JY, Xu J. Somatic gene copy number alterations in colorectal cancer: new quest for cancer drivers and biomarkers. Oncogene. Epub 2015 August 10.

22. Kelland LR. Overcoming the immortality of tumour cells by telomere and telomerase based cancer therapeutics - current status and future prospects. Eur J Cancer. 2005;41(7):971-979.

23. Ruden M, Puri N. Novel anticancer therapeutics targeting telomerase. Cancer Treat Rev. 2013;39(5):444-456.

24. Forsyth NR, Wright WE, Shay JW. Telomerase and differentiation in multicellular organisms: turn it off, turn it on, and turn it off again Differentiation. 2002;69(4-5):188-197.

25. Li Y, Tergaonkar V. Noncanonical functions of telomerase: implications in telomerase-targeted cancer therapies. Cancer Res. 2014;74(6): 1639-1644.

26. Sahin E, Colla S, Liesa M, et al. Telomere dysfunction induces metabolic and mitochondrial compromise. Nature. 2011;470(7334):359-365.

27. Nogueira V, Park Y, Chen CC, et al. Akt determines replicative senescence and oxidative or oncogenic premature senescence and sensitizes cells to oxidative apoptosis. Cancer Cell. 2008;14(6):458-470.

28. Liu JY, Qian D, He LR, et al. PinX1 suppresses bladder urothelial carcinoma cell proliferation via the inhibition of telomerase activity and p16/cyclin D1 pathway. Mol Cancer. 2013;12(1):148.

29. Zhang R, Zhao J, Wang X, Wang LL, Xu J, Song C. PinX1 without the G-patch motif suppresses proliferation, induces senescence, but does not inhibit telomerase activity in colorectal cancer SW480 cells. Oncol Rep. 2014;32(1):286-292.

30. Hahn WC, Meyerson M. Telomerase activation, cellular immortalization and cancer. Ann Med. 2001;33(2):123-129.

31. Falandry C, Bonnefoy M, Freyer G, Gilson E. Biology of cancer and aging: a complex association with cellular senescence. J Clin Oncol. 2014;32(24):2604-2610.

32. Wong KK, Chang S, Weiler SR, et al. Telomere dysfunction impairs DNA repair and enhances sensitivity to ionizing radiation. Nat Genet. 2000;26(1):85-88. 


\section{Supplementary materials}

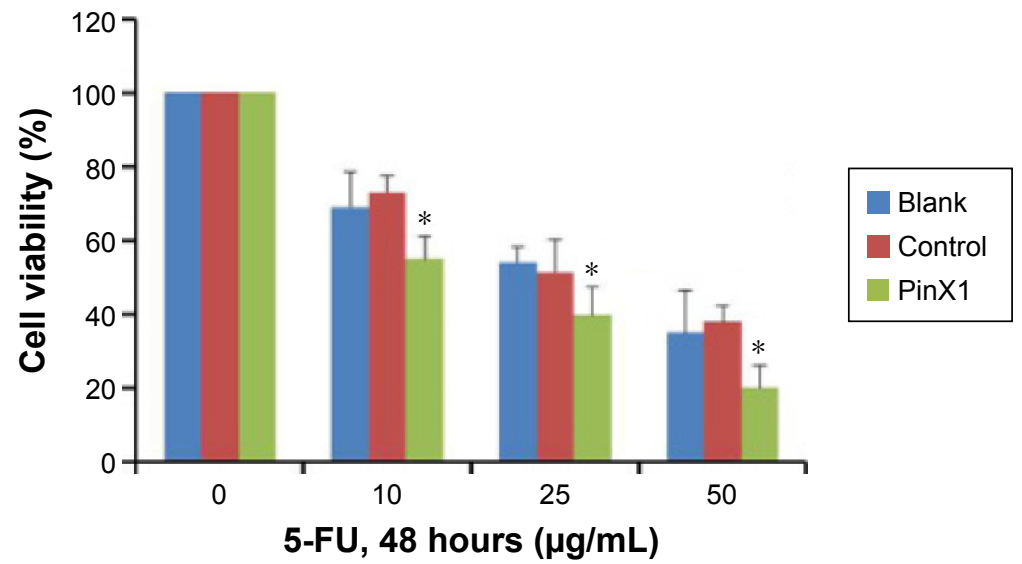

Figure SI Ectopic overexpression of PinXI enhances colorectal carcinoma cells' chemosensitivity to 5-fluorouracil (5-FU).

Notes: SWIII6-PinXI and corresponding control cells (blank and vector control) were treated with 5-FU for 48 hours at the indicated concentration. The cell viabilities were detected by 3-(4, 5-dimethylthiazol-2-yl)-2, 5-diphenyl tetrazolium bromide assay. Data represent the mean \pm standard error derived from three individual experiments with triplicate wells $(* P<0.05, P$-value was according to Student's $t$-test).
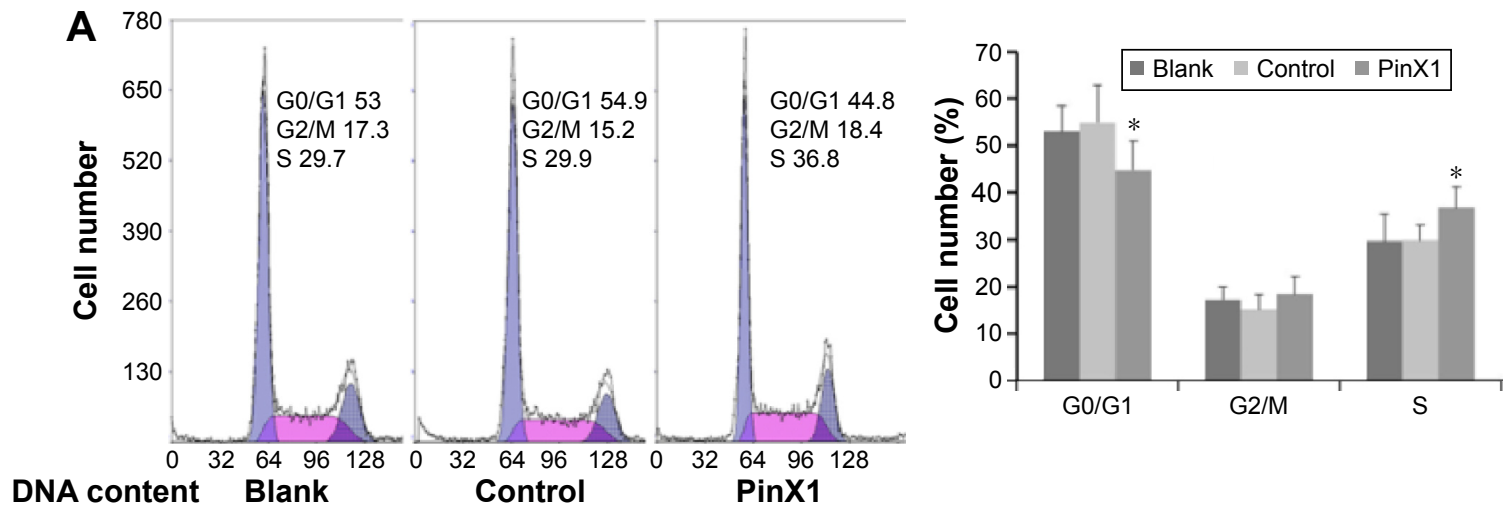

\section{DNA content Blank}

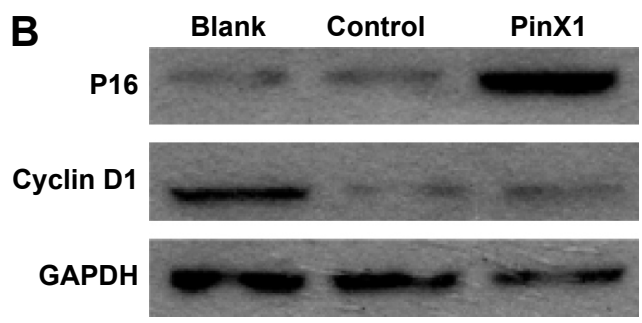

Figure S2 PinXI regulates GI/S phase transition of the cell cycle.

Notes: (A) Upregulation of PinXI expression in SWIII6 cells increased the proportion of cells in the G0/GI phase and decreased those in the S phase. The percentage of cell numbers was shown as the mean \pm standard error from three independent experiments ( $* P<0.05, P$-value was according to Student's $t$-test). (B) Overexpression of PinXI upregulated PI6 expression and downregulated Cyclin DI expression detected by Western blot. 
A

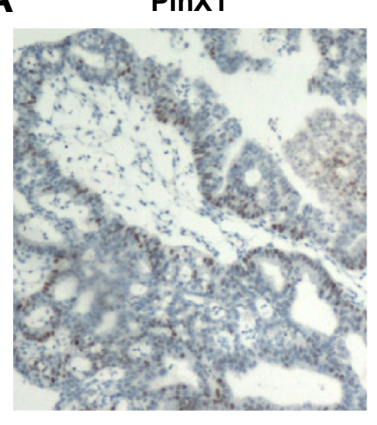

TERT

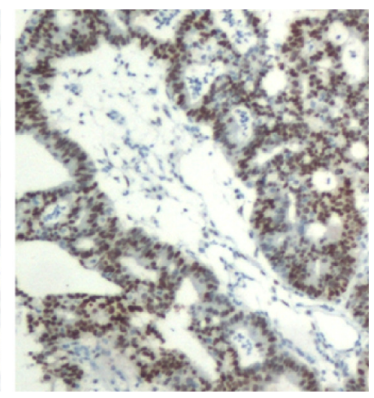

B

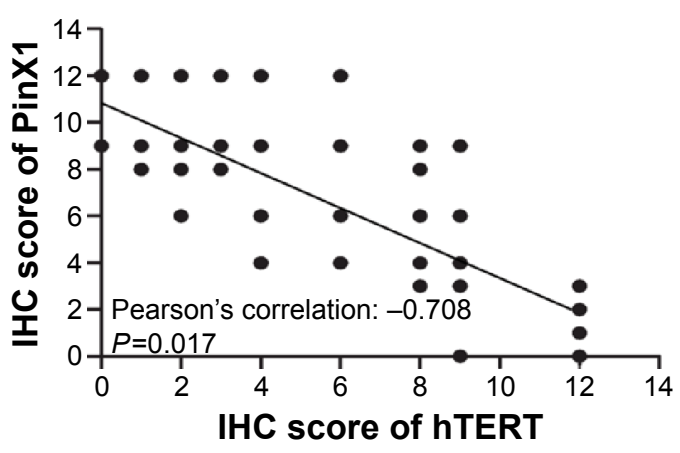

C

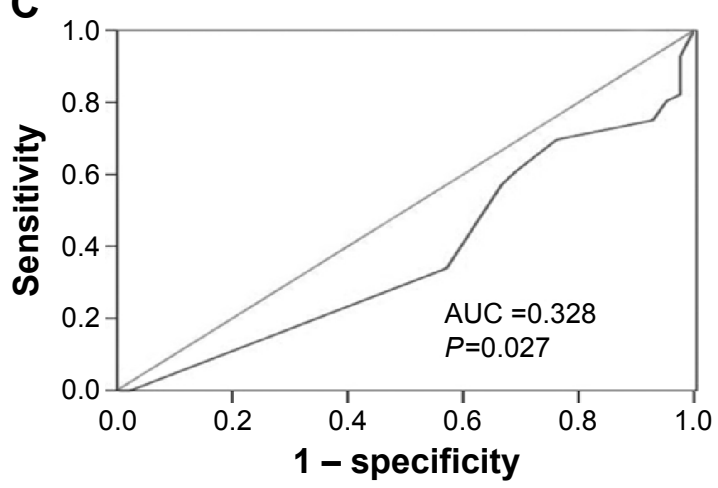

Figure S3 Correlation between the expression of PinXI and hTERT in CRC tissues.

Notes: (A) Representative PinXI and hTERT expression in CRC tissues determined by IHC. (B) Relationship of PinXI and hTERT expression in tumors. Herein, for evaluation of PinXI and hTERT staining, a semiquantitative scoring criterion was used, in which both staining intensity and positive areas were recorded. A staining index (values $0-12$ ) obtained as the intensity of positive staining (week, I; moderate, 2; strong, 3 ) and the proportion of immune-positive cells of interest ( $0 \%, 0$; < I0\%, I; $10 \%-50 \%$, $2 ; 51 \%-80 \%, 3 ;>80 \%, 4)$ were calculated. (C) The predictive value of PinXI expression regarding hTERT expression in CRC tissues. Receiver operation characteristic curve analysis was employed to determine the specificity and sensitivity of PinXI expression regarding hTERT expression (AUC $=0.328, P=0.027)$.

Abbreviations: AUC, area under the curve; CRC, colorectal carcinoma; hTERT, human telomerase reverse transcriptase; IHC, immunohistochemistry.

\section{Publish your work in this journal}

OncoTargets and Therapy is an international, peer-reviewed, open access journal focusing on the pathological basis of all cancers, potential targets for therapy and treatment protocols employed to improve the management of cancer patients. The journal also focuses on the impact of management programs and new therapeutic agents and protocols on

\section{Dovepress}

patient perspectives such as quality of life, adherence and satisfaction. The manuscript management system is completely online and includes a very quick and fair peer-review system, which is all easy to use. Visit http://www.dovepress.com/testimonials.php to read real quotes from published authors. 